\title{
THE OPERATIONAL CALCULUS OF LEGENDRE TRANSFORMS*
}

\section{Br R. V. ChURCHIL}

1. Introduction. The sequence of numbers $f(n)$ defined by the equation

$$
\left.f(n)=\int_{-1}^{1} F(x) P_{n}(x) d x \quad n=0,1,2, \cdots\right),
$$

where $P_{n}(x)$ denotes the Legendre polynomial of degree $n$, is the Legendre transform of the function $F(x)$. The integral transformation here will be represented by the symbol $T\{F(x)\}$. For functions $F(x)$ satisfying well-known conditions on the interval $-1 \leqq x \leqq 1$ the inverse of this transformation is represented by the expansion of $F(x)$ in series of the Legendre polynomials,

$$
F(x)=\sum_{n=0}^{\infty}\left(n+\frac{1}{2}\right) f(n) P_{n}(x)=T^{-1}\{f(n)\} \quad(-1<x<1) .
$$

Let $R[F]$ denote the differential form

$$
R[F(x)]=\frac{d}{d x}\left[\left(1-x^{2}\right) \frac{d}{d x} F(x)\right] .
$$

When the integral $T\{R[F]\}$ is integrated successively by parts and $-n(n+1) P_{n}(x)$ is substituted for $R\left[P_{n}(x)\right]$ in accordance with Legendre's differential equation, the following result is easily obtained.

ThEOREM 1: Let $F(x)$ denote a function that satisfies these conditions: $F^{\prime}(x)$ is continuous and $F^{\prime \prime}(x)$ is bounded and integrable over each interval interior to the interval $-1<x<1 ; T\{F(x)\}$ exists and

$$
\lim _{x \rightarrow \pm 1}\left(1-x^{2}\right) F(x)=\lim _{x \rightarrow \pm 1}\left(1-x^{2}\right) F^{\prime}(x)=0 .
$$

Then $T\left\{R\left[F^{\prime}(x)\right]\right\}$ exists and

$$
T\{R[F(x)]\}=-n(n+1) f(n) \quad(n=0,1,2, \cdots) .
$$

Formula (4) represents the basic operational property of the Legendre transformation $T$ under which the differential operation $R[F]$ defined by equation (3) is replaced by the algebraic operation $-n(n+1) f(n)$.

The established forms of operational calculus for solving problems in differential equations are based on integral transformations of Fourier type, transformations whose kernels are exponential functions or linear combinations of such functions. These transformations consist of the various Laplace and Fourier integral transformations. Transforms of the other types, including Legendre transforms [1], have been recognized as bases for other forms of operational calculus $[2,3,4]$, but to date the operational methods have not been developed beyond the stage of applying the basic operational property corresponding to formula (4) and the application of the inverse transformation. The type of

* Sponsored by the Office of Ordnance Research, U. S. Army, under Contract DA-20-018ORD-12916. 
boundary value problem that can be reduced by an integral transformation is of course governed by the kernel and the interval of integration [5].

A convolution property, one that gives directly the image of the operation of taking the product of the transforms of two functions, is now known for Legendre transforms [6]. Additional operational properties will be noted here and a short table of Legendre transforms will be developed. The operational calculus will then be illustrated by applying it to some classical boundary value problems in partial differential equations.

The application to the third boundary value problem for the potential inside a sphere leads to a simple expression for the solution of that problem in terms of the solution of a corresponding Dirichlet problem. This formula, together with the accompanying extension of the Poisson integral formula to the problem of the third kind, should be known; but a search of the literature and inquiry among colleagues has not yet revealed these formulas.

2. Operational properties. If each of the functions $R[F(x)]$ and $F(x)$ satisfy the sufficient conditions stated for the validity of formula (4) then the transform of the iterated differential form $R[R[F]]$ can be written at once as

$$
T\left\{R^{2}[F(x)]\right\}=n^{2}(n+1)^{2} f(n) .
$$

Similarly for iterations of higher order.

The substitution of $\left(n+\frac{1}{2}\right)^{2}-1 / 4$ for $n(n+1)$ in formula (4) leads to this modification of that operational property:

$$
\left(n+\frac{1}{2}\right)^{2} f(n)=T\{1 / 4 F(x)-R[F(x)]\} .
$$

It should be noted that under the substitution $x=\cos \theta(0 \leqq \theta \leqq \pi)$ our transformation

$$
T\{F(\cos \theta)\}=\int_{0}^{\pi} F(\cos \theta) P_{n}(\cos \theta) \sin \theta d \theta=f(n)
$$

ransforms the differential form

$$
R[F(\cos \theta)]=\frac{1}{\sin \theta} \frac{d}{d \theta}\left[\sin \theta \frac{d}{d \theta} F(\cos \theta)\right]
$$

nt o $-n(n+1) f(n)$, according to formula (4).

Consider now the transform of the function $R^{-1}[F]$, where $R^{-1}$ is the inverse iof the differential operator $R$. Let $Y(x)$ denote the function $R^{-1}[F(x)]$; then $t Y(x)$ is a solution of the differential equation

$$
R[Y(x)]=F(x) .
$$

Suppose that $F(x)$ is a function of bounded variation on each interval $|x| \leqq\left|x_{1}\right|<1$, and that

$$
\int_{-1}^{1} F(x) d x=0
$$


that is, $f(0)=0$. The first integral of equation (8),

$$
\left(1-x^{2}\right) Y^{\prime}(x)=\int_{-1}^{x} F(t) d t
$$

is then a continuous function of $x(|x| \leqq 1)$ with limit zero as $x \rightarrow \pm 1$. The second integral

$$
Y(x)=\int_{0}^{x} \frac{1}{1-s^{2}} \int_{-1}^{s} F(t) d t d s+C=R^{-1}[F],
$$

where $C$ is an arbitrary constant, is continuous when $|x|<1$ and it is easy to show that $\left(1-x^{2}\right) Y(x)$ vanishes as $x \rightarrow \pm 1$; in fact $|Y(x)|$ is of the order of $\left(1-x^{2}\right)^{-k}$ for each positive constant $k<1$ as $x \rightarrow \pm 1$ and hence $T\{Y\}$ exists. According to Theorem 1 and equation (8) then

$$
T\{R[Y]\}=-n(n+1) T\{Y\}=f(n) ;
$$

thus

$$
T\left\{R^{-1}[F]\right\}=-\frac{f(n)}{n(n+1)} \quad(n=1,2, \cdots) .
$$

The value of the transform of $R^{-1}[F]$ at $n=0$ depends on the value assigned to the constant $C$; if $F(x)$ is an odd function the value is easily shown to be $2 C$. The operational property concerning $R^{-1}$ can be stated as follows.

Theorem 2: Let $F(x)$ denote a function of bounded variation in each subinterval of the interval $-1<x<1$ and let $f(0)=0$. Then $f(n)$ exists and for each constant $\mathrm{C}$,

$$
\begin{array}{r}
T^{-1}\left\{\frac{-f(n)}{n(n+1)}\right\}=R^{-1}[F(x)]=\int_{0}^{x} \frac{1}{1-s^{2}} \int_{-1}^{s} F(t) d t d s+C \\
\quad(n=1,2, \cdots) .
\end{array}
$$

The convolution property can be stated as follows [6].

Theorem 3: Let $F(x)$ and $G(x)$ denote bounded integrable functions on the interval $-1 \leqq x \leqq 1$. Then the product $f(n) g(n)$ of their Legendre transforms is the transform of the function $H(x)$; that is,

$$
T^{-1}\{f(n) g(n)\}=H(x)
$$

where $H(x)$ is described by any one of these formulas:

$$
H(\cos \theta)=1 / \pi \int_{0}^{\pi} F\left(\cos \theta^{\prime}\right) \sin \theta^{\prime} \int_{0}^{\pi} G(\cos \lambda) d \phi d \theta^{\prime}
$$

where

$\cos \lambda=\cos \theta \cos \theta^{\prime}+\sin \theta \sin \theta^{\prime} \cos \phi ;$

$$
H(\cos \theta)
$$

$$
=1 / \pi \int_{0}^{\pi / 2} \sin \phi \int_{0}^{2 \pi} F\left[\sin \phi \sin \left(\beta-\frac{1}{2} \theta\right)\right] G\left[\sin \phi \sin \left(\beta+\frac{1}{2} \theta\right)\right] d \beta d \phi ;
$$




$$
\begin{aligned}
& H(x) \\
& \quad=1 / \pi \iint_{\boldsymbol{E}_{x}} F(y) G(z)\left(1-x^{2}-y^{2}-z^{2}+2 x y z\right)^{-1 / 2} d y d z
\end{aligned}
$$

where, for each fixed $x(-1<x<1), E_{x}$ is the region interior to the ellipse

$$
y^{2}+z^{2}-2 x y z=1-x^{2} \text {. }
$$

Formula (14) has a geometrical interpretation. Let $(r, \phi, \theta)$ denote spherical polar coordinates. If $P$ denotes any point $(1,0, \theta)$ on the boundary of a hemispherical surface of unit radius and $P^{\prime}$ any point $\left(1, \phi, \theta^{\prime}\right)$ on that surface, then according to the cosine law for spherical triangles $\lambda$ is the are $P P^{\prime}$ of a great circle. If $S$ denotes the hemisphere $r=1,0 \leqq \theta^{\prime} \leqq \pi, 0 \leqq \phi \leqq \pi$ then formula (14) can be written

$$
H(\cos \theta)=1 / \pi \iint_{\mathcal{S}} F\left(\cos \theta^{\prime}\right) G(\cos \lambda) d S
$$

thus, $H(\cos \theta)$ is twice the mean value of $F\left(\cos \theta^{\prime}\right) G(\cos \lambda)$ over the hemisphere. For $\theta=0$ and $\theta=\pi$ formula (14) reduces to

$$
H(1)=\int_{-1}^{1} F(t) G(t) d t, \quad H(-1)=\int_{-1}^{1} F(t) G(-t) d t,
$$

respectively. In view of formula (2) and the fact that $P_{n}(1)=1$ the first of equations (18) is seen to be the Parseval relation for the orthogonal set of functions $P_{n}(x)$.

TheOREM 4: If $T\{F(x)\}$ and $T\{G(x)\}$ exist then

$$
T\left\{C_{1} F(x)+C_{2} G(x)\right\}=C_{1} f(n)+C_{2} g(n),
$$

where $C_{1}$ and $C_{2}$ are constants; also

$$
T\{F(-x)\}=(-1)^{n} f(n) .
$$

These are obvious properties of the transformation since $T$ is clearly linear and since $P_{n}(-x)=(-1)^{n} P_{n}(x)$. When $G(x)=1$ then $g(n)=0(n=1,2, \cdots)$ and $g(0)=2$; according to equation (19) then, if $C$ is a constant,

$$
\begin{aligned}
T\{F(x)+C\} & =f(n) & \text { when } n=1,2, \cdots, \\
& =f(0)+2 C & \text { when } n=0 .
\end{aligned}
$$

The images under $T$ of the operations of differentiation and indefinite integration involve finite differences. Let $F(x)$ be a sectionally continuous function on the interval $-1 \leqq x \leqq 1$ and let $G(x)$ denote the continuous function

$$
G(x)=\int_{-1}^{x} F(t) d t
$$

Then

$$
f(n)=\int_{-1}^{1} G^{\prime}(x) P_{n}(x) d x=G(1)-\int_{-1}^{1} G(x) P_{n}^{\prime}(x) d x
$$


and it follows from a differential recurrence relation for $P_{n}(x)$ that

$$
\begin{aligned}
f(n-1)-f(n+1) & =\int_{-1}^{1} G(x)\left[P_{n+1}^{\prime}(x)-P_{n-1}^{\prime}(x)\right] d x \\
& =(2 n+1) g(n) \quad(n=1,2, \cdots) ;
\end{aligned}
$$

also, $f(0)=G(1)$ and since $P_{1}(x)=x, f(1)=G(1)-g(0)$ so that

$$
f(0)-f(1)=g(0) \text {. }
$$

The solution of the difference equation (21) for $g(n)$ and $f(n)$ in turn leads to these conclusions:

THEOREM 5: If $F(x)$ is sectionally continous then

$$
T\left\{\int_{-1}^{x} F(t) d t\right\}=\frac{f(n-1)-f(n+1)}{2 n+1}
$$

where, for $n=0, f(n-1)$ is to be replaced by $f(0)$. If $G(x)$ is continuous and $G^{\prime}(x)$ sectionally continuous and if $G(-1)=0$ then

$$
\begin{aligned}
T\left\{G^{\prime}(x)\right\}= & G(1)-(2 n-1) g(n-1)-(2 n-5) g(n-3)-\cdots-g(0) \\
& (n=1,3, \cdots), \\
& =G(1)-(2 n-1) g(n-1)-(2 n-5) g(n-3) \\
-\cdots-3 g(1) & (n=2,4, \cdots), \\
=G(1) & (n=0) .
\end{aligned}
$$

Other recurrence formulas for $P_{n}(x)$ lead to operational properties of the transforms, but none seem simple or promising.

3. Transforms of particular functions. A short table of Legendre transforms is presented here. Some methods of computing transforms in the table will now be indicated.

It follows at once from the orthogonality properties of the Legendre polynomials that when $F(x)=P_{m}(x)(m=0,1,2, \cdots)$ then

$$
f(n)=0(n \neq m), f(m)=1 /\left(m+\frac{1}{2}\right) .
$$

From the well-known representation of the function $G(x)=x^{m}$ in a finite series of Legendre polynomials of degree $m$ and lower it follows that $g(n)=0$ when $n>m$. The formula for $g(n)$ when $n \leqq m$ is not simple (cf. [7]). If $F(x)$ is any polynomial of degree $m$ then $f(n)=0$ when $n>m$.

The function $F(x)=\log (1-x)$ satisfies the condition $R[F]=-1$, where $R$ is the differential operator involved in Theorem 1 . But $F^{\prime}(x)$ does not satisfy the limit condition at $x=1$ in that theorem. Integration by parts, however, gives the equation

$T\{R[F]\}=-(1+x) P_{n}(x)||_{-1}^{1}-\left(1-x^{2}\right) \log (1-x) P_{n}^{\prime}(x)||_{-1}^{1}-n(n+1) f(n) ;$ that is,

$$
T\{-1\}=-2-n(n+1) f(n)=0 \quad(n=1,2, \cdots) .
$$


R. v. CHURCHILL

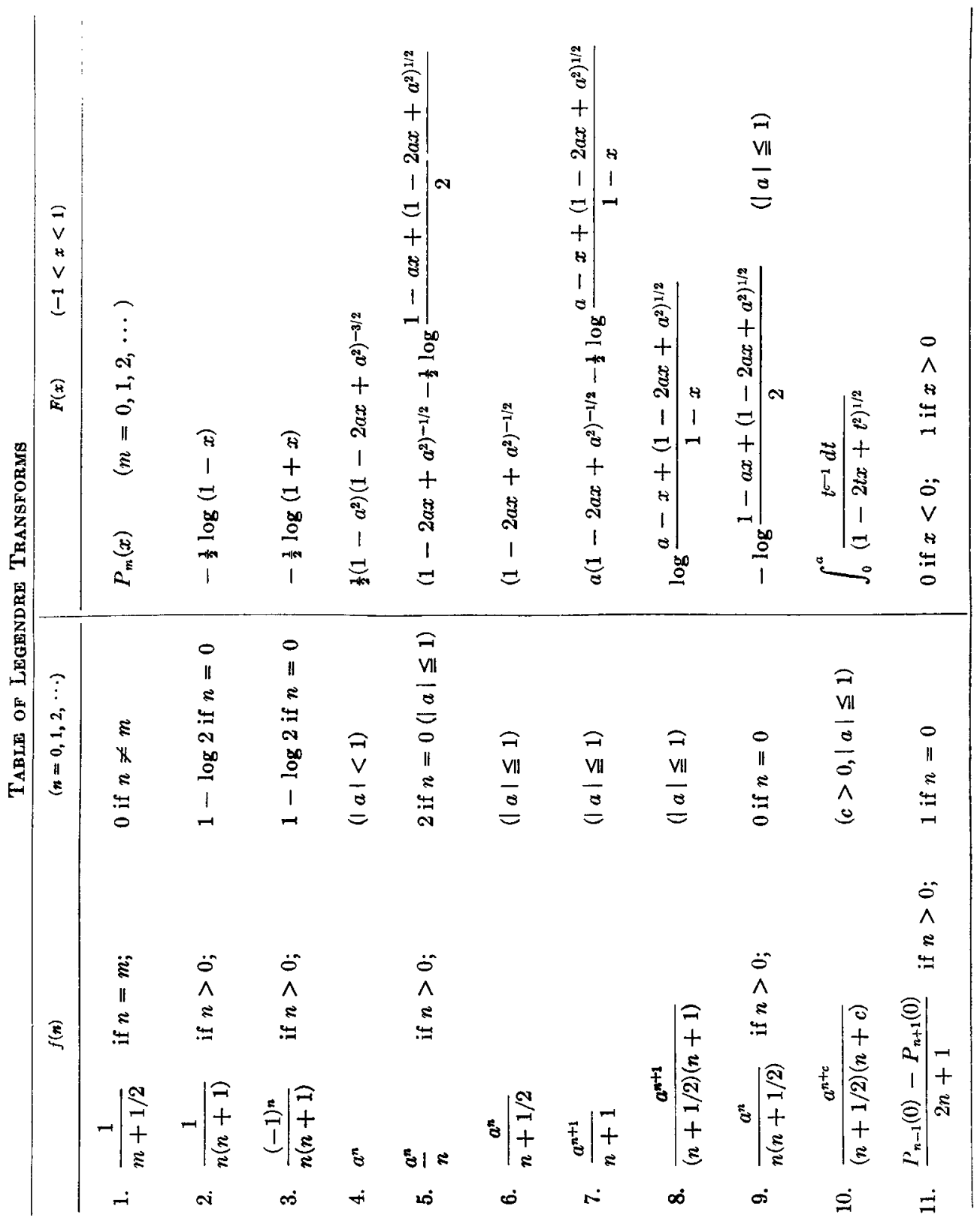


Direct integration gives $f(0)=2 \log 2-2$; hence

$$
\begin{aligned}
T\{\log (1-x)\} & =-2 / n(n+1) & & (n>0), \\
& =2 \log 2-2 & & (n=0) .
\end{aligned}
$$

The transform of $\log (1+x)$ follows from Theorem 4 .

From the uniformly convergent expansion of the generating function

$$
\left(1-2 t x+t^{2}\right)^{-1}=\sum_{n=0}^{\infty} t^{n} P_{n}(x)
$$

it follows that

$$
\int_{-1}^{1}\left(1-2 t x+t^{2}\right)^{-\frac{1}{3}} P_{n}(x) d x=t^{n} /\left(n+\frac{1}{2}\right) \quad(|t|<1) .
$$

When $t>0$ the integrand here is a continuous function of $t$ and $x$ except at $x=t=1$ and it is dominated by the function $\left(1-x^{2}\right)^{-\frac{1}{2}}$. Hence the integral is uniformly convergent with respect to $t$ up to $t=1$ and it represents a continuous function of $t$ there. Thus the transformation is valid when $t=1$; that is,

$$
T\left\{(1-x)^{-\frac{1}{2}}\right\}=\sqrt{2} /\left(n+\frac{1}{2}\right) .
$$

The transform of $(1+x)^{-\frac{1}{3}}$ follows from Theorem 4 .

Differentiation of both members of equation (24) with respect to the parameter $t$ followed by multiplication by $t$ leads to the equation

$$
\frac{1}{2} T\left\{\left(1-2 t x+t^{2}\right)^{-3 / 2}\left(2 t x-2 t^{2}\right)\right\}=n t^{n} /\left(n+\frac{1}{2}\right) \quad(|t|<1) .
$$

Since $2 t x-2 t^{2}=-\left(1-2 t x+t^{2}\right)+1-t^{2}$ it follows with the aid of formula (24) that

$$
T\left\{\left(1-2 t x+t^{2}\right)^{-3 / 2}\right\}=2 t^{n} /\left(1-t^{2}\right) \quad(|t|<1) .
$$

A continuation of the process leads to a slightly involved expression for $T^{-1}\left\{n t^{n}\right\}$ when $|t|<1$.

The transformation

$$
T\left\{\log \frac{a-x+\left(1-2 a x+a^{2}\right)^{\frac{1}{2}}}{1-x}\right\}=\frac{a^{n+1}}{\left(n+\frac{1}{2}\right)(n+1)} \quad(|a| \leqq 1)
$$

can be verified by integrating both members of equation (24) with respect to $t$. From the partial fractions expansion of the coefficient of $a^{n+1}$ here and from formula (24) with $t=a(|a| \leqq 1)$ it follows that

$$
T\left\{\frac{2 a}{\left(1-2 a x+a^{2}\right)^{\frac{1}{2}}}-\log \frac{a-x+\left(1-2 a x+a^{2}\right)^{\frac{1}{2}}}{1-x}\right\}=\frac{2 a^{n+1}}{n+1} \quad(|a| \leqq 1) \text {. }
$$

From equation (24) it follows that

$$
\begin{aligned}
\int_{-1}^{1}\left[t^{-1}\left(1-2 t x+t^{2}\right)^{-1}-t^{-1}\right] P_{n}(x) d x & =t^{n-1} /\left(n+\frac{1}{2}\right) & (n=1,2, \cdots) \\
& =0 & (n=0),
\end{aligned}
$$


and the integration of this equation with respect to $t$ leads to the transformation

$$
\begin{array}{rlrl}
T\left\{-\log \frac{1-a x+\left(1-2 a x+a^{2}\right)^{\frac{1}{2}}}{2}\right\} & =\frac{a^{n}}{n\left(n+\frac{1}{2}\right)} \quad(n=1,2, \cdots ;|a| \leqq 1) \\
& =0 & \quad(n=0 ;|a| \leqq 1) .
\end{array}
$$

From the representation of the coefficient of $a^{n}$ here in partial fractions the inverse transform of $a^{n} / n$ follows with the aid of formula (24).

Transform No. 10 in the table can be found by multiplying equation (24) by $t^{c-1}$ and integrating with respect to $t$. Transforms of step functions, illustrated by No. 11 in the table, are easily written with the aid of a well-known integration formula for $P_{n}(x)$. Combinations and special cases of the transforms in the table, as well as applications of the operational properties of the preceding sections, lead to a considerable extension of the list of transforms.

4. Applications. Dirichlet problem for sphere. Let $V(r, \cos \theta)$ denote the potential function interior to the unit sphere when the potential on the surface $r=1$ is a prescribed function $F(\cos \theta)$ of the spherical coordinate $\theta$ only $(0 \leqq \theta \leqq \pi)$. The interior is free from sources so that $V$ satisfies Laplace's equation

$$
\nabla^{2} V=\frac{1}{r}(r V)_{r r}+\frac{1}{r^{2} \sin \theta}\left(\sin \theta V_{\theta}\right)_{\theta}=0
$$

when $r<1$. If $x=\cos \theta$ this equation can be written

$$
r(r V)_{r r}+\left[\left(1-x^{2}\right) V_{x}\right]_{x}=0
$$

The function $V$ is also bounded in the region and satisfies the boundary condition

$$
V(1, x)=F(x) \quad(-1 \leqq x \leqq 1) .
$$

To solve this boundary value problem formally by means of the operational calculus developed in the foregoing sections the problem is written in terms of the transforms

$$
v(r, n)=T\{V(r, x)\}, f(n)=T\{F(x)\} \quad(n=0,1,2, \cdots)
$$

with respect to the variable $x$. Let the operator $T$ be applied to the members of equation (26) and (27) and let the order of differentiating $V$ with respect to $r$ and then integrating with respect to $x$ be reversed. In view of the basic operational property (Theorem 1 ) it follows that $v(r, n)$ satisfies the conditions

$$
r d^{2}(r v) / d r^{2}-n(n+1) v=0, \quad v(1, n)=f(n)
$$

and $v(r, n)$ is bounded when $0 \leqq r \leqq 1$.

The solution of the simple problem in $v$ here is

$$
v(r, n)=f(n) r^{n}
$$

and the inversion formula (2) can be used to represent $V(r, x)$ by an infinite series in the functions $r^{n} P_{n}(x)$, the form of the solution that would be obtained by separating variables in the boundary value problem (26), (27). The function 
$V(r, x)$ can be written in closed form, however, with the aid of the convolution property (Theorem 3) and the inverse transform of $r^{n}$ (No. 4 of the table). According to formula (14) for the inverse transform of the product of two transforms the inverse transform of $v(r, n)$ is

$$
\begin{array}{r}
V(r, \cos \theta)=\frac{1-r^{2}}{2 \pi} \int_{0}^{\pi} F\left(\cos \theta^{\prime}\right) \sin \theta^{\prime} \int_{0}^{\pi}\left(1+r^{2}-2 r \cos \lambda\right)^{-3 / 2} d \phi d \theta^{\prime} \\
(r<1),
\end{array}
$$

where

$$
\cos \lambda=\cos \theta \cos \theta^{\prime}+\sin \theta \sin \theta^{\prime} \cos \phi .
$$

This is the well-known Poisson integral formula for the potential inside a sphere in this special case in which the potential is a function of $r$ and $\theta$ only.

When $F(x)$ is a linear combination of any of the functions listed among the first nine items of the table, then $f(n) r^{n}$ can be written as a linear combination of the transforms listed in the table and the function $V$ can be written in a simple form free from integrals.

In the corresponding problem for the potential $W(r, \cos \theta)$ in the region exterior to the sphere $r=1$, the solution of the transformed problem is

Hence

$$
w(r, n)=(1 / r) f(n)\left(1 / r^{n}\right) \quad(r \geqq 1) .
$$

$$
W(r, \cos \theta)=(1 / r) V(1 / r, \cos \theta)
$$

where $V$ denotes the above potential function for the interior region. The Poisson integral formula for the potential $W$ follows at once from formulas (29) and (28).

When the potential is not independent of the spherical coordinate $\phi$ the Laplacian involves a differential operator with respect to $\cos \theta(x=\cos \theta)$ that is more involved than the operator $R$ of equation (3) and the transformation $T$ does not eliminate derivatives with respect to $x$ from the Laplacian.

5. Neumann problem for sphere. Let $U(r, \cos \theta)$ denote the potential in the region interior to the sphere $r=1$ when the normal derivative is a prescribed function $F(\cos \theta)$, so that

$$
\nabla^{2} U=0(r<1), \quad U_{r}(1, x)=F(x),
$$

where $x=\cos \theta$, and $U$ is bounded when $0 \leqq r \leqq 1$. Then $u(r, n)$, the transform of $U(r, x)$, is bounded and formally satisfies the conditions

$$
r(r u)^{\prime \prime}-n(n+1) u=0, \quad u^{\prime}(1, n)=f(n) \quad(n=0,1,2, \cdots)
$$

where the primes denote differentiation with respect to $r$.

When $n=0$ the bounded solution of the differential equation here is $u(r, 0)=C$, where $C$ is a constant. Hence $f(0)=0$ if the boundary condition is to be satisfied; thus

$$
\int_{0}^{\pi} F(\cos \theta) \sin \theta d \theta=0
$$


that is, the mean value of $F(\cos \theta)$ over the surface is zero, a well-known necessary condition for the solution of the Neumann problem.

The transformed problem has the solution

$$
\begin{aligned}
u(r, n) & =f(n) r^{n} / n & (n=1,2, \cdots) \\
& =C & (n=0)
\end{aligned}
$$

where $C$ is an arbitrary constant. Thus the potential $U(r, x)$ is determined up to an additive constant. It can be expressed in a series of the functions $r^{n} P_{n}(x)$ with the aid of the inversion formula (2); but it can be written in the closed form

$$
\begin{aligned}
U(r, \cos \theta) & =\frac{1}{\pi} \int_{0}^{\pi} F\left(\cos \theta^{\prime}\right) \sin \theta^{\prime} \int_{0}^{\pi}\left\{\left(1+r^{2}-2 r \cos \lambda\right)^{-\frac{3}{3}}\right. \\
& \left.-\frac{1}{2} \log \left[1-r \cos \lambda+\left(1+r^{2}-2 r \cos \lambda\right)^{\frac{1}{3}}\right]\right\} d \phi^{\prime} d \theta^{\prime}+C,
\end{aligned}
$$

where

$$
\cos \lambda=\cos \theta \cos \theta^{\prime}+\sin \theta \sin \theta^{\prime} \cos \phi^{\prime},
$$

with the aid of the convolution formula (14) and transform No. 5 in the table.

Let $Q$ denote any point $\left(1, \phi^{\prime}, \theta^{\prime}\right)$ on the hemisphere $S, r=1,0 \leqq \phi^{\prime} \leqq \pi$, $0 \leqq \theta^{\prime} \leqq \pi$, and let $P$ denote any point $(r, 0, \theta)$ of the region bounded by the boundary circle of $S$. Then the above variable $\lambda$ represents the angle between the radii through $P$ and $Q$ and the length of the line segment $P Q$ is

$$
\rho=\left(1+r^{2}-2 r \cos \lambda\right)^{\frac{1}{2}} \text {. }
$$

With the aid of formula (17) for the convolution, an alternate form of equation (32) now can be written as

$$
U(r, \cos \theta)=\frac{1}{2 \pi} \iint_{s} F\left(\cos \theta^{\prime}\right)[(2 / \rho)-\log (1+\rho-r \cos \lambda)] d S+C .
$$

This is a known form [8] of the Poisson integral solution of the Neumann problem for the sphere.

From equation (31) it follows that

$$
r u^{\prime}(r, n)=f(n) r^{n}=v(r, n) \quad(n=1,2, \cdots),
$$

where $v(r, n)$ is the transform of the Dirichlet problem (26), (27), with the same function $F(x)$ there as here. Thus

$$
\begin{aligned}
u(r, n) & =\int_{0}^{r} v\left(r^{\prime}, n\right) d r^{\prime} / r^{\prime} & (n=1,2, \cdots), \\
& =C & (n=0)
\end{aligned}
$$

and the inverse transformation gives the expression

$$
\begin{aligned}
U(r, \cos \theta) & =\int_{0}^{r} V\left(r^{\prime}, \cos \theta\right) d r^{\prime} / r^{\prime}+C \\
& =\int_{0}^{1} V(r t, \cos \theta) d t / t+C
\end{aligned}
$$


for the solution of the Neumann problem (30) in terms of the solution of the Dirichlet problem (26), (27). This relationship between the solutions can be verified directly and it is not necessary to assume that the potential functions and the function $F$ are independent of the coordinate $\phi$.

6. Problem of third type for sphere. Now let $U(r, \cos \theta)$ represent the bounded potential function for the region interior to the sphere $r=1$ satisfying the mixed type of boundary condition at the surface. Specifically,

$$
\nabla^{2} U=0(r<1), \quad U_{r}(1, x)+(k+1) U(1, x)=F(x)
$$

where $x=\cos \theta$ and $k+1$ is a positive constant. The function $U$ can be interpreted as the steady-state temperatures at points in a solid sphere which is subjected to linear heat transfer at its surface into surroundings whose temperature is proportional to $F(\cos \theta)$.

The transform $u(r, n)$ of $U(r, x)$ is the bounded function that satisfies the conditions

$$
r(r u)^{\prime \prime}-n(n+1) u=0, \quad u^{\prime}(1, n)+(k+1) u(1, n)=f(n) ;
$$

hence

$$
u(r, n)=f(n) r^{n} /(n+k+1) \quad(0 \leqq r \leqq 1, k>-1) .
$$

It follows that

$$
r^{-k}\left(r^{k+1} u\right)^{\prime}=f(n) r^{n}=v(r, n),
$$

where $v(r, n)$ is the transform of the solution $V(r, x)$ of the Dirichlet problem (26), (27); therefore

$$
u(r, n)=\int_{0}^{r} v(s, n)(s / r)^{k} d s / r=\int_{0}^{1} v(r t, n) t^{k} d t .
$$

The inverse transformation then gives the formula

$$
U(r, \cos \theta)=\int_{0}^{1} V(r t, \cos \theta) t^{k} d t
$$

for the solution of problem (35) of the third type in terms of the solution of the Dirichlet problem (26), (27) with the same function $F(x)$.

A generalization of formula (37) can be verified easily. Let the above functions $U, V$ and $F$ be replaced here by $U(r, \phi, \theta), V(r, \phi, \theta)$ and $F(\phi, \theta)$. For the sake of simplicity assume that $F$ is a continuous function of its two variables.

Theorem 6: The potential function $U(r, \phi, \theta)$ of the third boundary value problem for the sphere,

$$
\nabla^{2} U=0(r<1), \quad U_{r}(1, \phi, \theta)+(k+1) U(1, \phi, \theta)=F(\phi, \theta)
$$

where $k>-1$ and constant and the function $F$ is continuous, is given in terms of the solution $V(r, \phi, \theta)$ of the Dirichlet problem

$$
\nabla^{2} V=0(r<1), \quad V(1, \phi, \theta)=F(\phi, \theta)
$$


by the formula

$$
U(r, \phi, \theta)=\int_{0}^{1} V(r t, \phi, \theta) t^{k} d t .
$$

The verification that the function $U$ given by formula (40) is harmonic in the region $r<1$ when $V$ is harmonic there is straightforward. To show that the function $U$ satisfies the boundary condition in problem (38) let $r t=s$ and note that

$$
r U_{r}(r, \phi, \theta)=\int_{0}^{1} V_{s}(s, \phi, \theta) t^{k+1} r d t=\int_{0}^{1} V t(s, \phi, \theta) t^{k+1} d t
$$

when $r<1$. Hence

$$
\begin{aligned}
& r U_{r}(r, \phi, \theta)+(k+1) U(r, \phi, \theta) \\
& =\int_{0}^{1}\left[t^{k+1} V_{t}(s, \phi, \theta)+(k+1) t^{k} V(s, \phi, \theta)\right] d t=V(r, \phi, \theta)(r<1) .
\end{aligned}
$$

It is known that the function $V$ is continuous in the region $r \leqq 1$ and it follows from formula (40) that $U$ is continuous there. According to equation (41) then $r U_{r}$ is also continuous there. When $r=1$ equation (41) reduces to the boundary condition in problem (38).

A Poisson integral formula for the solution of the third boundary value problem (38) follows at once by substituting the known Poisson integral that represents the solution $V$ of the Dirichlet problem into formula (40). Other forms of the Poisson integral formula for the special case (35) in which $U$ is independent of $\phi$ can be found from the formula (36) for $u(r, n)$ with the aid of the convolution property. For the inverse transform of $r^{n}(n+k+1)^{-1}$ can be found either from transforms No. 10 and No. 6 in the table or by integrating the transformation No. 4 after multiplying by $a^{k}$.

The relation (40) between the solutions of the two types of problems for the sphere is so simple that it should be known. Neither that relation nor a Poisson integral formula for the problem of the third kind for the sphere nor the corresponding results in logarithmic potential for the circle, which follow similarly from finite Fourier transformations, have been found yet in the literature.

If $Y(r, \cos \theta)$ denotes the potential in the exterior region $r>1$ for the third problem

$$
\nabla^{2} Y=0(r>1), \quad Y_{r}(1, x)-k Y(1, x)=-F(x)
$$

where $k>0$ here and constant and $x=\cos \theta$, the transform of $Y$ is easily found to be

$$
y(r, n)=(1 / r) f(n) r^{-n} /(n+k+1)=(1 / r) u((1 / r), n) \quad(r \geqq 1)
$$

where $u(r, n)$ is the transform (36) of the solution of the third problem (35) for the interior of the sphere. Hence

$$
Y(r, \cos \theta)=(1 / r) U(1 / r, \cos \theta) \quad(r>1) .
$$


Moreover, the procedure used to arrive at formula (37) is easily applied to the above formula for $y(r, n)$ to arrive at the formula

$$
Y(r, \cos \theta)=\int_{1}^{\infty} W(r t, \cos \theta) t^{-k-1} d t
$$

where $W(r, \cos \theta)$ is the potential (29) in the Dirichlet problem for the exterior region,

$$
\nabla^{2} W \neq 0(r>1), \quad W(1, \cos \theta)=F(\cos \theta) .
$$

The verification of formulas (43) and (44) shows that the potentials and the function $F$ involved need not be independent of the cooridnate $\phi$.

7. Distributed sources in sphere. As a final illustrative application of the transforms consider the problem

$$
\nabla^{2} V(r, x)=F(r, x)(r<1), \quad V(1, x)=0
$$

where $x=\cos \theta$. The transformed problem is

$$
r^{2} v^{\prime \prime}(r, n)+2 r v^{\prime}(r, n)-n(n+1) v(r, n)=r^{2} f(r, n), \quad v(1, n)=0 .
$$

Its solution can be written by elementary methods in the form

$$
\begin{aligned}
v(r, n)=\int_{0}^{1} \frac{(r s)^{n}}{2 n+1} f(s, n) s^{2} d s-\frac{1}{r} \int_{0}^{r} \frac{s^{n} r^{-n}}{2 n+1} f(s, n) s^{2} d s & \\
& -\int_{r}^{1} \frac{r^{n} s^{-n}}{2 n+1} f(s, n) s d s
\end{aligned}
$$

According to transform No. 6 in the table,

$$
T\{G(a, x)\}=a^{n} /(2 n+1) \quad(|a| \leqq 1)
$$

where

$$
G(a, x)=(1 / 2)\left(1+a^{2}-2 a x\right)^{-\frac{1}{2}} .
$$

If $H(a, s, x)$ denotes the convolution (Theorem 3 ) of the two functions $G(a, x)$ and $F(s, x)$ as functions of $x$ the formal inverse transformation of the members of equation (46) gives the formula

$$
V(r, x)=\int_{0}^{1} H(r s, s, x) s^{2} d s-\frac{1}{r} \int_{0}^{r} H\left(\frac{s}{r}, s, x\right) s^{2} d s-\int_{r}^{1} H\left(\frac{r}{s}, s, x\right) s d s
$$

for the solution of problem (45).

\section{REFERENCES}

1. Tranter, J. C., Integral Transforms in Mathematical Physics, Wiley, New York, 1951.

2. Sneddon, I. N., Fourier Transforms, McGraw-Hill, New York, 1951.

3. Burgat, P., "Résolution de problèmes aux limites au moyen de transformations fonctionnelles", J. de Math. Phys. Appl. 4, 146-152 (1953).

4. Sсотт, E. J., “Jacobi Transforms”, Quart. J. Math. (Oxford) 4, 36-40 (1953). 
5. Churchild, R. V., "Integral Transforms and Boundary Value Problems", Amer. Math. Monthly 69, 149-155 (1952).

6. Churchill, R. V., and Dolph, C. L., "Inverse Transforms of Products of Legendre Transforms", Proc. Amer. Math. Soc. 5, 93-100 (1954).

7. Churchill, R. V., Fourier Series and Boundary Value Problems, McGraw-Hill, New York, $1941 ; 188$.

8. Kellogg, O. D., Potential Theory, Springer, Berlin, 1929.

The University of Michigan

(Received September 8, 1953) 